\title{
Parcours politique d'une apparition
}

Le cas de Lourdes

\section{Élisabeth Claverie}

\section{OpenEdition}

Journals

Édition électronique

URL : http://journals.openedition.org/assr/21046

DOI : 10.4000/assr. 21046

ISSN : $1777-5825$

Éditeur

Éditions de l'EHESS

\section{Édition imprimée}

Date de publication : 1 janvier 2009

Pagination : 109-128

ISBN : 978-2-7132-2215-3

ISSN : 0335-5985

\section{Référence électronique}

Élisabeth Claverie, "Parcours politique d'une apparition », Archives de sciences sociales des religions [En ligne], 145 | janvier-mars 2009, mis en ligne le 01 janvier 2012, consulté le 19 avril 2019. URL : http:// journals.openedition.org/assr/21046 ; DOI : 10.4000/assr.21046 


\section{Élisabeth Claverie}

\section{Parcours politique d'une apparition Le cas de Lourdes}

\section{Les apparitions comme événement}

Dans certains cas, et vu par une sociologie ou ethnographie pragmatique, le statut de la croyance passe par une prise en compte de son caractère non immédiat, c'est-à-dire du caractère non automatique ou direct de sa réception par les individus ou les groupes, contrairement à ce que sous-entend le substantif «croyance» dans certaines expressions dont usent les sciences sociales : " être sous croyance ", "faire partie d'un système de croyances, de représentations ". Cette ethnographie critique tend à ameublir un peu les analyses qui donnent au terme un caractère totalement englobant, via les notions de culture ou de socialisation par exemple, pour saisir les acteurs au moment de leurs incertitudes et de leurs choix dans le cours d'action et laisser aux sciences sociales la faculté de repérer ces événements ou micro évènements dans leur dimension d'élaborations singulières et collectives, souvent conflictuelles. Apparaît ainsi le caractère pluriel, non consensuel, de ces élaborations, et la pluralité des facteurs qui entrent en cause. Il arrive en effet que ce qui vient se présenter à la reconnaissance ou à l'assentiment n'aille pas de soi, même lorsqu'il s'agit d'un objet religieux recensé comme connu et attesté ici (comme une Vierge apparaissante, toujours décrite ex-post, après sa reconnaissance stabilisée, dans la littérature). C'est notamment le passage de l'individuel au collectif qui peut quelquefois ne pas s'effectuer sans le recours à une chaîne de médiateurs favorables et défavorables, ou encore la prise en compte publique et collective de l'énonciation d'une expérience religieuse d'abord alléguée comme expérience intime ou personnelle. Cette ethnographie donne alors une place importante aux médiations empiriques qui viennent se placer entre l'objet de croyance et les acteurs. Elle regarde comment ceux-ci modèlent tel ou tel objet de croyance et les formes qu'il prend sous l'effet de ces diverses prises sociales souvent contradictoires entre elles. Elle le décrit comme absorbant en lui toute l'épaisseur des contradictions politiques, économiques et sociales du moment.

Dans cette perspective, le terme de «croyances» est considéré comme un "corpus " labile et toujours transformable, capable de venir à la rescousse de telle ou telle situation pour la louer ou la critiquer. Non pas depuis lui-même, 
mais dans un croisement entre sa structure ontologique et les prises qu'elle donne, ici et maintenant, à ses usages politiques actualisés. Ontologiquement, une Vierge-Apparition, du fait même de sa structure interne, vient toujours montrer ou dévoiler sa formule initiale qui consiste en l'affirmation de l'existence de contradictions : "il y a de la contradiction », elle affirme aussi que le réel n'est pas entièrement rabattu sur lui-même et sur ses déterminations actuelles et elle affirme un système ni symétrique ni clos des relations entre la vie et la mort. Politiquement, elle signifie, sans mots, par le seul fait d'apparaître à un être liminaire, tout le spectre des réalités d'une crise économique et sociale décrite par ses victimes. Elle donne des espoirs eschatologiques de renversements, une contre-culture, une critique des processus trop rapides de la modernisation là encore par ceux qui s'en estiment les victimes ${ }^{1}$, qui produisent alors leurs effets jusqu'à un certain point. Celui de la reprise en main par les dispositifs de paroles constitués sur une toute autre scène que la scène initiale d'apparition, une scène des pouvoirs, des controverses, des opinions contradictoires et de leurs représentants. En effet, ce trajet politique de la vision ne s'arrête pas à ce premier moment. Très vite, il change de mains. S'ensuivent des controverses pro et contra. Des causes adverses se constituent dont les fins s'éloignent des buts de la scène initiale avec leurs équipements argumentatifs et narratifs respectifs qui s'attachent à certains dispositifs d'expression (presse, partis, jeux d'opinions, théories de la connaissance, etc.).

En ce qui concerne Lourdes, mais c'est souvent le cas s'agissant des apparitions des $\mathrm{XIX}^{\mathrm{e}}$ et $\mathrm{XX}^{\mathrm{e}}$ siècles, on assiste très vite, même si les arguments critiques sont très présents et trouvent là de riches occasions de se développer, à l'expression d'une vision politique au moins conservatrice (prêches, journaux, rachat du site, mise à l'écart de B [Bernadette de Lourdes désormais B pour limiter le caractère trop puissamment évocateur de constructions idéologiques acquis par ce prénom dans l'historiographie de Lourdes]. Il ne s'agit plus alors, comme dans l'instant B, de critique des modalités locales de la modernisation, mais d'attaques de groupes politiques contre la modernité ${ }^{2}$. Ce qui n'est pas la même chose. C'est dans le processus de cette conversion d'une revendication à une autre (de la critique des formes que prend ici et maintenant la modernisation à une critique politique de principe de la modernité) que se tient la première phase des apparitions. Le point de vue que j'essaie de défendre suppose donc un petit déplacement : considérer les apparitions non comme le produit direct d'une culture religieuse et d'une situation sociale (ce qu'elles sont aussi), mais comme un événement qui produit lui-même une situation sociale se montrant dans sa réflexivité, ses mobilisations et leurs moyens. Le trouble qui survient permet en effet aux acteurs et aux sciences sociales d'avoir accès à des explicitations locales,

1. Voir, par analogie, les analyses de Karl Polanyi (1983).

2. Dans le cas de Lourdes, il s'agit des relais du parti ultra qui cherchait une revanche à la Révolution. 
tenues habituellement dans l'implicite. Bref, regarder les apparitions plutôt comme événement tourné vers un futur que comme produit social du passé. Afin de ne pas pré-qualifier ce qui s'est passé à Lourdes, je ne me préoccuperai pas de la différence entre visions, voyance et apparitions, les trois choses n'étant pas distinguées par les acteurs locaux dans la première phase du phénomène social qui m'intéresse ici, mais qui deviendra, par contre, essentiel dans les débats de qualifications de la période suivante, au moins pour certains acteurs.

\section{Les sources}

Je ne traiterai ici que des toutes premières séquences de la première phase de "l'affaire Lourdes » (février-juillet 1858), phase pendant laquelle il s'est agi d'identifier à la fois le type de mensonge de la voyante, son degré de crédibilité et l'identité de ce qui lui apparaissait. La bibliographie critique et le catalogue complet des sources d'archives n'ont pas été réalisés à ce jour. Actuellement, le corpus des sources provient principalement du récolement puis de la publication des sources d'état-civil, des interrogatoires et dépositions de témoins (obtenus par le commissaire de police, la gendarmerie, le préfet, le procureur), la correspondance officielle de l'administration civile et religieuse, les articles de presse locale et nationale et les enquêtes entreprises par plusieurs médecins, prêtres, amateurs divers, auprès de $\mathrm{B}$, de son entourage, des villageois de la bourgade. Ces sources ont été collationnées par l'abbé René Laurentin et par Dom Billet et publiées, mais ce travail n'a pas été complètement achevé ${ }^{3}$. D'autres sources sont conservées au couvent de Saint-Gildard, à Nevers, certaines sont mises en ligne, un inventaire est en cours. Il faut compter aussi nombre d'ouvrages littéraires rédigés après une visite sur les lieux, par des enquêteurs ecclésiastiques ${ }^{4}$, des écrivains aussi célèbres que Huysmans, Zola, Alexis Carel ou Franz Werfel ; mais sont surtout nombreuses les œuvres de médecins souvent engagés entre eux dans des controverses au sujet des "prétendues guérisons miraculeuses » et de l'estimation des effets de la suggestion (Charcot, 1897 ; Bertrin, 1905 ; Charuty, 1988, 1992). On dispose également aujourd'hui d'œuvres de sciences sociales sur Lourdes, notamment le très beau livre de l'historienne anglaise qui a renouvelé le sujet, Ruth Harris (2001).

3. R. Laurentin, Lourdes, Documents authentiques (LDA), 6 vol., 1956-1961, puis T. VII, par Dom Billet, pour les années 1862-1866. Laurentin a ensuite commencé, à travers la mise en œuvre d'une chronologie, une mise en récit appuyée sur ces mêmes sources, rendant explicitement « visible » son travail d'interprétation dans Lourdes, Histoire authentique (LHA), 6 vol., 1961-1964.

4. Notamment le premier d'entre eux, Henri Lasserre, préfacé par Pie IX : Notre-Dame de Lourdes, Paris, 1869. 


\section{Premières Séquences : revendiquer une élection}

Lorsque chacun des six «Voyants » de Medjugorje - cinq adolescents et un enfant de huit ans - annoncèrent à leurs familles qu'ils venaient de "voir la Vierge ", ou qu'une " chose ", « une forme ", « une lumière ", " une Dame ", " une petite fille ", leur était apparue, ils reçurent de leurs mères respectives une gifle. Il en fut de même pour B : fille de bientôt quatorze ans, elle reçut de sa mère, à la même annonce d'ailleurs faite par sa sœur, des coups de bâton. Plus tard dans la soirée, s'adoucissant, la mère expliqua à sa fille qu'elle avait fait un rêve et lui interdit formellement de retourner sur les lieux. Elle considérait qu'elle avait déjà assez à faire avec les autorités (c'est-à-dire, pour elle, tout le monde) pour se faire davantage remarquer. Il s'agissait par ces gestes de ramener ces enfants " à la réalité ", au tangible, de reprendre le contrôle de leurs émotions et de leur sens de la hiérarchie, de restaurer leur sens moral (on ne doit pas mentir) et de les socialiser à la mesure de ce qu'il y avait à voir et à ne pas voir, ou à dire publiquement en tout cas. Une note de nature différente, moins directement explicite, venait généralement surligner ces sages considérations : il s'agissait de rendre ces enfants sensibles au discernement social, et à la compréhension de qui pouvait prétendre à ce type de vue, le cas échéant. Après qu'ils eurent donné la gifle et les coups de bâton, leurs parents leur avaient fait comprendre qu'il n'y avait absolument aucune raison pour que ce soient eux, enfants très ordinaires à tous égards, voire socialement marginaux et misérables, qui reçoivent ce privilège, si privilège il $\mathrm{y}$ avait. Aucune raison pour que ne soient pas choisis leurs parents, par exemple, comme dans le cas de Medjugorje, ou quelque belle demoiselle de la congrégation des Enfants de Marie, s'agissant de Lourdes. "Pourquoi vous? » leur demanda-t-on avant tout. Comment justifiaientils, eux, leur demandait-on constamment, leurs prétentions d'accès à un autre espace de perception? De sortie d'une perception commune ? À de telles expériences ? À une telle élection?

C'est qu'en effet, sans une assise de justifications très étayée, revendiquer un privilège, une élection, est un acte social des plus risqués. Cela relève de la gageure lorsque l'on est une fille de quatorze ans, couverte de vermine, louée sans gages dans la montagne, vêtue misérablement, ne mangeant pas à sa faim, illettrée et ne parlant pas français, dont les parents ont dans la bourgade la réputation usuelle des pauvres : paresseux, ivrognes, instables. Et ceux-là étaient logés dans un taudis, par charité, avec leur marmaille. Révélés crûment, on retrouve toutes ces qualifications, tous ces jugements omniprésents dans les enquêtes de voisinage, dans les rapports des autorités municipales et départementales. Bref, cette fille et sa famille se placent tout en bas de la cascade des mépris locaux comme, en général, des mépris les mieux partagés dans la France de 1858.

Ajoutée à ces obstacles sociaux, l'annonce de B se heurta à une difficulté d'un autre ordre : la difficulté d'ajustement entre plusieurs cadres d'expérience et leurs modalités respectives d'expression. 


\section{Parler à la première personne}

La gifle et le bâton portaient aussi un autre type d'accusation : ils pointaient une absence de compétence à discerner la différence entre sémantique des légendes et récits faisant intervenir un être surnaturel, tenus pour vrais, mais d'un "vrai - faux, pas toujours vrai, pas toujours faux »d'un certain type, les modes et techniques de narrativité dans lesquels ils sont exprimés et les moyens narratifs de la vie ordinaire, et les rencontres qu'on y fait et qu'on relate. S'introduire à la première personne (indexicalité), au moyen de la langue de l'expérience en propre, dans ce qui est un lieu de réalité floue, d'habitude commandé par des énonciations indirectes (ragots, contes récités dans certaines situations, images, tableaux, statues, textes lus pendant l'office), demandait une série de vérifications pour être reçu. Rendre présent ici et maintenant, attesté par la parole d'un être spécialement non fiable, ce qui circulait d'habitude dans les récits de rencontres avec des êtres d'autres mondes, mais dans des récits pourvus de marqueurs linguistiques spécifiques pour en indiquer le genre, était abusif, socialement, cognitivement. Prenaient-ils les autres pour des idiots?

\section{Questions de qualifications}

Il semble que la grande difficulté de la situation de la toute première phase des « histoires d'apparition », l'annonce de sa vision par celui qui n'est pas encore un voyant, mais encore un membre de la communauté classé et perçu sous d'autres rapports, concerne le passage (en public) d'un cadre référentiel de connaissances dispersées, issues de récits d'autorité (tradition), agencées empiriquement dans de multiples dispositifs pratiques, à un récit en première personne sans supports ou relais pratiques. En effet, les divers récits autorisés en circulation, quel que soit le principe de cette autorisation, récits éprouvés de tous types, ne sont pas envisagés par leurs usagers comme appartenant à la catégorie des expériences directes d'un membre quelconque de la communauté locale. Ces récits, prônes, leçons de catéchisme, images variées, statues, contes, font partie d'une catégorie langagière mobilisée dans certains actes par certaines personnes, et par soi-même dans certains dispositifs cultuels ou sociaux, mais pas ainsi, dans une scène où les personnes sont brutalement invitées « à sauter dans l'image ", à importer des parties de cette image ici, parmi nous, dans l'espace ordinaire (et inversement), et à y faire pénétrer par un médiateur médiocre une entité de l'autre monde chez soi, au village. Parvenir à rendre poreux ces deux ensembles, que ce soit pour les attester ou les contredire, est donc pour les acteurs, une entreprise de longue haleine (Latour, 1990). On s'intéressera ici aux difficultés et aux supports que rencontrent sur place, mais aussi "plus loin » et ailleurs, les annonces locales de relations avec "le surnaturel ", lorsqu'elles ne viennent pas corroborer de façon consensuelle une fonction rituelle attendue, instituée, ou légitimer une 
spécialité professionnelle. Dans le cas des «apparitions de la Vierge » contemporaines, en effet, les « voyants » sont immédiatement requis de produire publiquement un discours sur leurs expériences et, surtout, de les rendre sensibles, visibles, et partageables, jusqu'à ce que d'autres relais s'engagent dans cette cause ou dans la cause adverse, ces derniers visant à les discréditer selon tous rapports ou sous certains rapports.

Par anticipation donc, et sur plusieurs plans, le récit proféré de la scène d'apparition ouvre sur un risque de désordre: dans une société d'interconnaissance avec ses savoirs et ses jugements, surgit un être non identifié, sans papiers, ni catégorie identifiée, non répertorié. Cet être non identifié a été vu, dans le cas de Lourdes, par une seule personne, une mineure, plutôt sale, d'aspect fragile et replié. Son groupe familial est dépendant de la charité publique, ou objet, quand cela se présente, d'une exploitation sans phrases. Bref, elle est de peu de poids social, et, comme on sait, dans la plupart des cas de revendication d'une rencontre surnaturelle, l'énonciateur d'une telle nouvelle se trouve rapidement rejeté.

\section{Péripéties d'une revendication}

Cette revendication d'une brèche dans les choses telles qu'elles sont, cette surprise qui lui est faite, est assumée par Bernadette. Mais cette acceptation revient à quitter (au moins symboliquement) le réalisme, la nécessité, la contingence, l'ordre naturalisé des choses sociales et politiques. La gifle et le bâton sont là pour arrêter ce mouvement. Mais l'énonciatrice résiste dans des scènes qui deviendront emblématiques de cette résistance et de cette affirmation paradoxale - scène de l'interrogatoire du commissaire de police et de la tenue courageuse et probe de $\mathrm{B}$ face à un représentant de l'État retors et menteur - scènes qui trouvent leur paradigme et leur répertoire d'action dans l'imagerie de Jeanne d'Arc et de sa posture lors de son procès. Est figurée ici un dispositif d'asymétrie politique, mais aussi la réalisation d'une promesse eschatologique du renversement de cette asymétrie et de la formule sociale qui la sous-tend. L'énonciatrice est dotée d'une volonté et veut " que passe » l'énonciation de cet acte de rupture, l'énonciation de cette ouverture (pour elle). L'assertion de B, donnée comme le fait d'une expérience soudaine qui l'a surprise, dans laquelle elle n'est pour rien, qu'elle n'a ni provoquée ni attendue, ni méritée (dans une économie politique dominante du mérite), mais qu'elle rend publique, va ainsi se diffuser de proche en proche et s'insérer comme une provocation dans le tissu même de l'ordre local - la famille, les voisins, le curé, le commissaire de police, le préfet, le corps médical -, et projeter plus loin encore le récit par le biais de médiations plus puissantes : la presse locale sera rapidement prévenue, et bientôt la presse nationale. D'autres enjeux dans d'autres scènes politiques, économiques et sociales seront mis en branle. 


\section{Circulation du récit et publicité de l'apparition}

B, qui se déchaussait pour rejoindre sa sœur et une amie de l'autre côté du Gave où celles-ci vaquaient déjà à leur tâche de ramassage de bois et d'os, entendit un souffle de vent. Alertée, elle leva la tête et vit en face d'elle, dans une anfractuosité de la barrière de roche, une « douce lumière sortir d'une niche de la grotte. L'instant d'après une enfant belle et souriante apparut qui semblait lui faire un signe d'approcher ». Effrayée, continue le récit, B sortit son chapelet de sa poche, mais l'enfant en face d'elle la précéda dans son geste et fit un signe de croix. B refit son geste en l'imitant. Aucune parole ne fut prononcée. C'est avec cette scène, racontée par les auteurs à partir des déclarations de B lors de ses interrogatoires et de ses écrits autographes ${ }^{5}$, que commence le récit de la série de ses dix-huit apparitions. La première eut lieu le 11 février 1858 et la dernière, cinq mois plus tard, le 16 juillet 1858.

Il y avait eu des témoins. Une scène d'apparition en comporte toujours. Cette présence de tiers modifia la scène initiale de la rencontre visuelle entre l'apparition et celle qui la vit. Scène privée, colloque amoureux, elle devint, presque d'emblée, publique. Ces témoins, en l'occurrence la sœur de B et l'amie qui les avait accompagnées, ne virent pas B voir, puisqu'à ce moment-là, elles jouaient pour se réchauffer à danser près de la grotte, ne donnant pas de signification particulière au fait de la voir agenouillée de l'autre coté du ruisseau. À ses compagnes "Vous n'avez rien vu ? ", elles la pressèrent de leur dire ce qui se passait. Ce qu'elle fit. Elle ne fit pas cependant le récit décrit plus haut, établi plus tard, lorsque "l'apparition donna son nom » et que la série donna rétrospectivement un sens à chaque occurrence des visites surnaturelles antérieures. Elle dit à sa sœur Toinette qu'elle avait vu "Quelque chose, Cela » (aquerò en patois bigourdan), la jeune sœur le répéta à sa mère. Celle-ci en parla, dans la soirée, à sa parente et logeuse, qui habitait au-dessus. Le lendemain, 12 février, Toinette en parla aux élèves de sa classe, la classe des indigents de l'Hospice de Lourdes tenu par les Filles de la Charité de Nevers, et B à une religieuse de l'ouvroir, ces propos étant traduits en français par une compagne. Un espace public était constitué. Lorsque B retourna pour la seconde fois à la Grotte, le 14 février 1858, une douzaine de filles de la classe gratuite de l'Hospice la suivaient, et une seconde apparition eut lieu qui engagea un premier rituel d'identification. Les fillettes avaient préparé de l'eau bénite que B lança sur l'entité, aux fins de savoir «si c'était bon ou si c'était mauvais ». Ce geste ne fit pas fuir l'apparition. La même scène, dans les mêmes conditions, et avec les mêmes paroles prononcées, eut lieu à Medjugorje.

Le lendemain, en confession, $B$ décrivit en patois bigourdan la scène au vicaire de la paroisse, l'abbé Pomian : "J'ai vu quelque chose de blanc ayant la forme

5. Il y a sept récits autographes, tous rédigés à l'hospice de Lourdes entre 1861 et 1866. Ils sont conservés au couvent de Saint-Gildard à Nevers. 
d'une dame ». Le vicaire parla de la chose le soir même au curé, celui-ci lui répondit, peu intéressé, qu'il fallait attendre. Des rumeurs se répandaient en ville et dans la campagne alentour, parmi lesquelles un bruit commençait à se faire jour : ce qui se montrait à $B$ était certainement l'âme d'une jeune fille décédée récemment pieusement, Elisa Latapie, qui avait présidé la congrégation locale des Enfants de Marie.

\section{Qu'est-ce que c'est ? Problèmes d'identité}

La seconde séquence de cette première phase montre la tension entre la volonté de $\mathrm{B}$ de maintenir l'entité dans une certaine indétermination (Aquero) et celle des autorités exigeant la détermination de son nom. Quand on lui demandait de spécifier à quoi ressemblait «Cela », B répondait que c'était " une dame plutôt petite que grande» (quelquefois une petite fille), "vêtue d'une robe blanche et d'une ceinture bleue, une rose jaune ou dorée posée sur chacun de ses pieds ", mais qu'elle ne savait pas qui était cette Dame ou plutôt, elle y insistait ${ }^{6}$, cette petite Demoiselle. C'est ce qu'elle répondit de manière constante au commissaire de police Jacomet lors de son premier interrogatoire, le 21 février 1858. Dans son rapport, celui-ci transformera pourtant immédiatement la qualification "Cela» en "la Vierge ». Nom qui circulait avec d'autres parmi les rumeurs (telle ou telle âme, un mort, une fée, un esprit, la Vierge). Ce qui empêchait de reconnaître absolument la Vierge, c'est que cette Dame ne tenait pas d'enfant Jésus dans les bras. Elle avait l'air de venir en propre, ce qui était une nouveauté. Cette seconde séquence va impliquer de nouveaux acteurs, intéressés selon des lignes diverses à cette auto-nomination surveillée qui trouvera des alliés pour que soit prononcé plutôt tel nom que tel autre. Ces nouveaux acteurs sont liés à des réseaux sociaux plus longs et lointains, débordant les hiérarchies locales (le département, le pays, le ministère, la faculté de médecine, l'évêque). Cette exigence de nomination fut le point d'appui de la première étape de reprise en main par les autorités, le début d'une stabilisation de " ce qui arrive ", mais organisée selon des dispositifs échappant aux premiers acteurs, B et ses compagnes. Pour l'instant, en effet, s'établissait une première forme de fixation de la scène, une forme locale avec ses dévots et ses sceptiques. Des fidèles avaient construit un autel devant la grotte, qui comprenait fleurs, statue, bougies et dons en argent. Le maire et le préfet le firent détruire par les gendarmes et saisir les dons.

La question du nom, donc, devenait centrale pour toutes les autorités, maire, préfet, procureur et curé. Il fallait sortir de l'indétermination catégorielle pour constituer une cause et une saisie par l'opinion. C'est-à-dire objectiver un corps universellement reconnaissable, renvoyant à des références compréhensibles

6. Ruth Harris (1999) insiste beaucoup sur ce point, avec raison. 
au-delà de la sphère locale, à un système de classifications et d'opinions sociopolitiques. Ce corps, désormais politique, pourra alors donner prise à des controverses sur une nouvelle scène. Pour cela, il fallait aussi un corps identifié, nommé, fixé, assignable.

La chose était devenue un problème de police. Le caractère de publicité des visions de $\mathrm{B}$, avec des groupes amassés autour de la grotte, des questions discutées par les citoyens de la ville entre eux, bref, des troubles de l'ordre public, entraîna la constitution d'une configuration spécifique : l'enquête de type judiciaire. Celle-ci diligenta à son tour une enquête policière, administrative, médicale, mobilisant des témoins et des experts, le curé, le vicaire, l'évêque, la mère supérieure de l'hospice, les sœurs de l'ouvroir. Le préfet, le maire, le procureur général, le procureur, le commissaire de police, les gendarmes, le garde champêtre et bientôt les médecins, locaux et venant de villes plus importantes, furent mobilisés de part et d'autre de la barre. Des renseignements furent recoupés. Parallèlement à cette enquête, s'en déroulait une autre, parmi les habitants de la ville et de la campagne alentour, faite de supputations, de comparaisons d'indices, de commentaires, de ouï-dire : " mais qui apparaissait à B pouvait-on la croire ? ". Ces deux formes d'enquêtes ne portaient pas sur la même personne, pour les uns, l'objet de l'enquête portait plutôt sur "la prétendue voyante », pour les autres, sur l'entité.

\section{Bernadette, objet d'enquête}

B était devenue l'objet d'une surveillance policière intense, un objet d'enquête. Elle fut d'abord longuement interrogée le 21 février par le commissaire de police, Jacomet, qui lui interdit de retourner à la grotte sous peine de sanction. Elle ne promit rien. Jacomet dépêchera des gendarmes pour la surveiller et surveiller le site et enverra son rapport au préfet. Il se rendra lui même sur place muni d'un calepin, à partir du 25 mars, date à laquelle l'apparition dévoilera son nom : «Je suis l'immaculée conception».

«... Le nombre des visiteurs est immense (Pau, Tarbes, Bagnères), on y vient des départements voisins. Chacun emporte une bouteille d'eau de la fontaine miraculeuse. En ce qui me concerne, j'ai surveillé la grotte cette nuit, d'une manière toute particulière, et j'étais sur les lieux bien avant l'arrivée de la visionnaire pour y constater ses faits et gestes, dont j'ai l'honneur de vous entretenir plus haut ${ }^{7}$. "

Suivi, deux jours plus tard du rapport suivant :

"Monsieur le Préfet, vous aurez l'aspect de la Grotte pendant, vers mi journée du jeudi 25 mars, quand vous saurez qu'à dix heures du soir, cinquante-deux cierges y brûlaient encore à la fois ; vous aurez aussi une certaine idée du nombre immense des

7. 25 mars 1858, Rapport Jacomet, LDA, VI, p. 42. 
visiteurs quand j'aurai eu l'honneur de vous dire que les offrandes de la journée se sont élevées au chiffre énorme de 61 francs presque toutes en monnaie de billon - je garantis ce chiffre (biffé : j'ai compté) ${ }^{8}$."

Puis, du même :

"Elle a été suivie pas à pas par le Dr Dozous qui a constamment tenu les yeux sur elle. J'ignore le résultat de ces observations ${ }^{9}$... »

Ce même jour, le maire, Lacadé, écrit au préfet une lettre qui montre que, même s'il n'y a pas de désordre cultuel (prier Dieu, et non pas on ne sait quoi), le désordre tient dans l'affluence :

«... Elle a prié Dieu environ trois quarts d'heure et puis, elle s'est retirée. L'ordre le plus parfait n'a cessé de régner. L'affluence des curieux a été considérable le 4 et le

5 du courant. On y remarquait et on y a compté :

- étrangers : 4238

- de la ville : 4822

- Total : 9 060. $>$

\section{Une âme du purgatoire}

Lors de la troisième apparition fut expérimentée une nouvelle hypothèse. Une jeune fille de Lourdes, couturière, Antoinette Peyret, membre de la congrégation des Enfants de Marie et une de ses amies, Madame Milhet, ancienne servante ayant épousé son maître, femme riche désormais, ayant entendu en ville les rumeurs, firent venir B chez Madame Milhet et l'interrogèrent sur sa vision. On doit préciser ici que la mère de $\mathrm{B}$ travaillait souvent « à la journée » chez cette madame Milhet. Et l'on voit s'ouvrir un instant d'inversion sociale. Les deux femmes prièrent la jeune fille de pouvoir l'accompagner à la grotte le lendemain pour demander à Aquero son nom et ses désirs, se rendant chez ses parents pour une demande de permission. L'une et l'autre pensaient qu'il s'agissait en fait de l'âme d'Elisa Latapie, récemment décédée. C'est, en effet, Antoinette Peyret qui avait confectionné le vêtement de congréganiste dans lequel Elisa avait été ensevelie et elle le reconnaissait dans les descriptions de B :

«J'avais pensé que, puisqu'une Dame apparaissait avec une robe blanche et ceinture bleue, c'était notre supérieure des Enfants de Marie, Elisa Latapie, morte depuis peu de mois. Cette congrégation était sous l'invocation de l'Immaculée conception depuis 1841, date de la fondation. Je me disais donc : c'est le costume des Enfants de Marie: bande bleue, nouée devant - et à l'heure de la consécration, nous avions le chapelet au bras, au poignet, pour les enterrements aussi ${ }^{10}$. »

Les tantes de $\mathrm{B}$ pensaient la même chose : ce que voyait leur nièce, c'était une âme du purgatoire, mais elles pensaient plutôt « à l'âme de quelqu'un de la

8. 27 mars 1858, Rapport Jacomet, LDA, VI, p. 43.

9. 7 avril 1858, Rapport du commissaire Jacomet au Préfet Massy, LDA, VI, p. 44.

10. Antoinette Peyret, interrogatoire du 3 ou 4 juin 1878, LHA, 5, p. 210. 
famille ». C'est ainsi que les deux femmes, Milhet et Peyret, partent ce 18 février, très tôt le matin, munies d'une écritoire, d'une plume et de papier pris au greffe, le frère de Mademoiselle Peyret étant greffier. Pour s'assurer de ce que voulait Elisa, il fallait lui demander de le noter par écrit. Arrivées à la grotte, elles commencent à réciter le chapelet quand Bernadette voit soudain paraître «l'enfant blanche ». Alors Mme Peyret tire de son sac la feuille blanche, l'écritoire, la plume et un encrier et tend ce matériel à B avec cette requête : "Demande à la Dame ce qu'elle veut et qu'elle le mette par écrit ». On sent ici l'influence de la vogue des réunions spirites et le dispositif d'écriture qu'il requiert souvent pour que les esprits (lettrés) se nomment. B s'exécute : "Voulez-vous avoir, demande-t-elle, la volonté de mettre votre nom par écrit? " La Dame, relata B, se met à rire et ajoute en mépris de ces exigences : "Ce n'est pas nécessaire ». Puis, en regardant B, elle lui demande en patois : "Voulez-vous avoir la grâce de venir ici pendant quinze jours ? " Stupéfaite d'une telle déférence et de ce vouvoiement, Bernadette acquiesce, et la Dame lui annonce alors : " Je ne vous promets pas de vous rendre heureuse en ce monde, mais dans l'autre". Lorsqu'elle revient à elle, ses compagnes lui demandent si elle a reconnu Elisa Latapie. Sur la réponse négative de B, Madame Milhet change d'alternative: «Et si c'était la Sainte Vierge ?»

Par cette scène, culture orale (en patois) et culture écrite (en français) sont manifestées dans toute la tension que leur inégal maniement produit dans la région, et se révèle l'anxiété engendrée par les avancées de la culture écrite, de l'école, du français étayés par la force de leurs appuis institutionnels. De retour à Lourdes, Madame Milhet informe la mère de $\mathrm{B}$ de sa décision de prendre celleci chez elle. C'est que les notables de la ville, s'avisant soudain de ses conditions de vie, veulent extirper B du cachot, tout en y laissant sa famille. L'élection spirituelle qui semble être la sienne méritant peut-être, considèrent-ils, quelques réajustements sociaux. La famille n'accepte pas. C'est ce même jour que paraît une brève dans le journal de l'arrondissement d'Argelès, le journal local paraissant chaque jeudi, jour de marché à Lourdes, Le Lavedan, journal républicain : «Une jeune fille que tout fait supposer atteinte de catalepsie fixe, pique la curiosité de la population de Lourdes... » On commence à entrevoir deux types de diagnostics à Lourdes, bien résumés par le procureur impérial dans un rapport au Procureur général du $1^{\text {er }}$ mars $1858^{11}$ :

"Suivant le petit nombre, qui prétend avoir pour soi le bon sens, la raison et la science, Bernarde Soubirous est atteinte d'une maladie mentale connue : elle est en proie à des hallucinations ; mais le nombre est très grand, au contraire, et va s'accroissant, de gens de toute condition sociale, qui la croient en communication directe avec la Divinité. »

11. Premier Rapport du procureur impérial de Lourdes Dutour au Procureur général Falconnet, $L D A, 1$, p. 175. 


\section{La mise en forme esthétique : l'icône et sa destruction}

L'enquête des autorités à l'égard de $\mathrm{B}$ visait à identifier la nature de son mensonge. Supercherie, maladie, hystérie, jeu ? Le médiateur de cette enquête sera le visage de $\mathrm{B}$, son corps, ses attitudes. Ils devinrent le lieu privilégié de l'investigation policière, médicale, mais aussi dévotionnelle. Ce que la jeune fille expérimentait était inscrit sur son visage, considérait-on, et il fallait, à côté des interrogatoires directs, l'observer aux fins de préciser, de compléter un diagnostic. Il en était de même pour la foule de ses suiveurs, mais selon une interprétation qui n'avait pas pour centre une lecture clinique, mais une lecture mystique. Ce visage "illuminé », cette pieuse attitude, cette prostration, cette pâleur de cire, ces sourires et ces larmes étaient une attestation de ce qu'elle voyait, la preuve de la vérité de ses dires, inscrite sur elle par l'action de la vision. Son visage était devenu l'écran sur lequel on pouvait lire le reflet de ce que faisait et disait l'entité, très probablement, la Vierge. Il était une interface entre la voyante et sa vision, et montrait en quelque sorte les deux ensemble, dans leur dialogue. Cette médiation du corps de la voyante, de sa posture animée par l'entité, sera pour les suiveurs une des médiations de "l'entrée dans le tableau ", à laquelle nous faisions allusion plus haut. Le tableau admettant la porosité des mondes. Cette posture sera pour les autres l'entrée dans le tableau clinique.

On présentera ici deux scènes d'apparition contrastées, la quatrième et la neuvième. L'une, la quatrième, installe la scène publique de médiation, en formalise l'icône. Elle dresse la scène in vivo, réplique de la dévotion " bonne et vraie ". On sait que B fut la première sainte photographiée, mimant en studio, à la demande des photographes, certaines scènes d'apparition. La seconde, la neuvième, viendra défaire cette scène, montrant le degré de dépendance de l'admission aux «bonnes formes".

\section{Installation iconographique}

Au cours de la quatrième apparition, le vendredi 19 février 1858, à six heures du matin, la tante et marraine de B, Bernarde ${ }^{12}$, fille aînée de la fratrie maternelle, le côté dominant de la parentèle de $\mathrm{B}$, donne à celle-ci, qui était agenouillée le chapelet à la main et entamait les prières du rosaire, un cierge de la congrégation des Enfants de Marie. Bernarde allume ce cierge et vient se placer immédiatement à la droite de sa nièce. Dans chaque scène d'apparition désormais, Bernadette conservera ce cierge de congréganiste, cette posture et cet équipement, avec sa tante placée à sa droite, en organisatrice de la cérémonie. Ces deux gestes d'intervention et d'installation rituelles, liés à la volonté de manifestation d'une

12. Les filles ainées de la famille sont nommées Bernarde. C’est aussi le véritable prénom de Bernadette, qui s’appelle «Bernarde » comme sa tante aînée. 
aura de pureté, gestes effectués par la tante et marraine de Bernadette sont adressés de manière significative au public lourdais, puisque la tante Bernarde fut longtemps discréditée comme "fille-mère ». Elle se maria nettement après la naissance de son enfant, avec le père de celui-ci. Partant, elle avait été exclue, socialement et moralement, de la congrégation des Enfants de Marie, ce qui était considéré comme une honte publique. L'appartenance aux Enfants de Marie était un signe très recherché d'honorabilité par la population féminine. Les deux tantes de B, Bernarde et Basile, en avaient été exclues pour les mêmes raisons de maternité hors mariage. On essaya donc de les faire sortir de l'image, de la proximité de la Voyante. On lit ainsi dans une Lettre du Père Cros, un des grands enquêteurs littéraires de Lourdes, corrigeant le récit d'Estrade, témoin visuel qui plaçait, dans ses souvenirs, Basile et Bernarde à proximité de B, pour la remplacer par Lucile, « la bonne tante ». Mais c'est bien Bernarde qui accompagne toujours B :

« La tante Basile était une fille-mère notoire. Elle ne figura jamais à côté de Bernadette aux apparitions. Elle fut chassée des Enfant de Marie. Lucile, enfant de Marie, est la tante notoirement honorable. La marraine s'était, elle aussi, trop hâtée de devenir mère ; mais le fait était déjà vieux ; elle assista Bernadette à la grotte : ce qui n'empêcha pas le scandale (pharisaïque sans doute) de bien des gens que cette seule considération éloigna longtemps de la grotte - ceci soit dit pour votre instruction! » ${ }^{13}$

Au troisième "Je vous salue Marie », les traits de B, toujours agenouillée, son cierge dans une main et son chapelet dans l'autre, changent. Elle devient très pâle, comme de la cire diront les témoins, "ses yeux ne vacillent pas ", elle sourit doucement et salue l'apparition :

"Elle salue avec les mains et la tête : c'était un plaisir de la voir, comme si toute sa vie elle n'avait fait autre chose que d'apprendre à faire ces saluts. Je ne savais faire que la regarder ${ }^{14}$.»

De multiples témoignages disent la même chose. "Elle se meurt » crie quelqu'un dans la foule et cette "petite mort» est souvent soulignée par les témoins. Beaucoup pleurent. La référence à cet état d'extase, de ravissement, deviendra constante. Comme le montre ce témoignage tardif (1878) qui s'applique à décrire, par contraste avec les signes de présence lisibles sur le visage de Bernadette, leur absence quand B se rend à la Grotte et que la Vierge n'y apparaît pas :

«Dans la période du 2 au 25 mars, la petite bergère se rendit à peu près tous les jours à la Grotte. Elle y priait, elle buvait à la source comme tout le monde, mais sa figure ne reflétait pas les clartés de la céleste vision, ne subissait plus de transformation ${ }^{15}$. »

Ainsi, ce qu'observent les autorités, c'est une jeune fille qui regarde quelque chose, avec autour d'elle une foule qui la regarde, ressent, et montre, à son tour,

13. Lettre du P. Cros au P. Brucker, LHA, VI, p. 235.

14. Témoignage de Josèphe Barinque, LHA, p. 69-70.

15. Estrade. Mémoire de Septembre 1878, Minute, LHA, VI, p. 63. 
des émotions au contact visuel du visage de B. Lorsque cette petite mort prend fin, B se ranime, retrouve ses couleurs et, assistée de la foule, redescend vers chez elle. Pendant le trajet, les gens l'interrogent, mais elle répond seulement qu'Aquero n'a rien dit, qu'elle lui a seulement souri. On ne sait pas encore si c'est Elisa Latapie ou la Vierge que voit B. De nombreux témoins, lorsque interrogés par les autorités, racontent la beauté, la douceur et le sentiment de complétude offerts par cette scène, malgré l'indétermination du nom de la vision, la Vierge commençant à être, pourtant, la meilleure candidate. Ce même jour, un peu plus tard, un voisin, Barinque, époux d'un témoin oculaire de l'apparition du jour, lui attache une médaille miraculeuse de la rue du Bac autour du cou, portant la gravure de la Vierge de l'Immaculée conception et l'invocation qui lui est consacrée ( O Marie, conçue sans péché, priez pour nous qui avons recours à vous »).

\section{Mimétisme}

Plus tard, cette attitude (agenouillée, pâle et souriante, les yeux levés, un cierge dans une main, un chapelet dans l'autre, puis laissant voir sur son visage les péripéties de la rencontre) sera systématisée par B, qui se livrera à sa propre imitation, imitant la Vierge. Ce fut le cas notamment à propos de l'apparition «centrale» du 25 mars, celle au cours de laquelle l'apparition livrera son nom ( "Je suis l'Immaculée Conception »). Le récit initial de B viendra donner un sens particulier à cette imitation : lorsque, au cours de la première apparition, elle avait mentionné que l'apparition lui avait montré comment elle devait se servir de son chapelet (prônant la dévotion au rosaire), B la mima. Cette première relation mimétique, puis la réplique de ce mime par $\mathrm{B}$, mimant à nouveau ce premier mime devant un public, par sa mise en scène dialogique, fut un des dispositifs clés permettant l'appropriation par les personnes, ici et maintenant, de cette apparition-là. Plus tard, à Lourdes même, ou lorsqu'elle l'eut quitté, on demanda souvent à $\mathrm{B}$ de se mimer elle-même telle qu'elle était lors de cette apparition du 25 mars. Par exemple :

«J'ai vu et interrogé des personnes devant lesquelles Bernadette avait reproduit cette scène en imitant les gestes de la Sainte Vierge, toutes ont été fortement impressionnées par la grâce de Bernadette étendant ses mains comme la Sainte Vierge, les inclinant, puis les relevant pour les joindre, élevant les yeux au ciel ; il s'échappait de toute sa personne je ne sais quoi de céleste qui faisait dire à tous ceux qui étaient les heureux témoins de cette scène - il n'y a pas à douter : elle a vu ce qu'elle reproduit. Tous déclarent que ces choses ne peuvent s'exprimer telles qu'elles ont été ressenties. Il y avait sur la figure de Bernadette, un reflet de beauté qu'ils sont impuissants à traduire, mais qu'ils n'oublieront jamais ${ }^{16}$.»

16. R. Quidarré, déposition, 25 février 1894, LHA, VI, p. 79. 
"Bernadette (imitant la scène du 25 mars) eut un geste qui causa une émotion indicible ${ }^{17}$."

Ou encore, au couvent de Nevers :

"Le dimanche après son arrivée [à Nevers le 8 juillet 1866], on nous fit raconter par $\mathrm{B}$, les apparitions... Je ne puis encore penser sans une très vive émotion à l'expression de son regard, de son geste, de sa voix, quand elle a dit en faisant le geste de la Sainte Vierge : Je suis l'immaculée conception ${ }^{18}$. »

Le curé de Lourdes, Peyramale, lui demande aussi de se produire :

«M. Peyramale lui dit : - Allons ! Fais comme la Sainte Vierge lorsqu'elle t'a dit "Je suis l'immaculée conception !". Je suis dans l'impossibilité de reproduire ce que j'a vu tellement j'ai été touché ; c'est un souvenir que je n'oublierai jamais ${ }^{19}$. "

\section{Contre-épreuve : une scène bestiale}

Cette composition iconographique, cette parfaite image de dévotion, va bientôt se trouver bouleversée. On attire ici l'attention sur l'importance de la forme de la scène dans le processus d'attestation publique dont $\mathrm{B}$, ou telle autre «voyante », est le centre.

Le 25 février (neuvième apparition), la foule qui entoure B n'a plus la même composition sociale qu'au début. De nombreux notables, favorables, ironiques ou indécis, sont présents dans un coin du site sur lequel se massait le peuple de Lourdes et des environs. Il y avait ce jour-là trois cent cinquante personnes. On vit soudain B adopter un étrange comportement. Jusqu'à présent, on l'a vu, elle présentait dans ses extases, malgré sa pâleur de cire, l'image d'une dévotion réglée, belle à voir, statique, le visage "illuminé d'une joie intérieure ». Mais ce jour-là, à la grande gêne de ses proches et de tous, elle montre subitement aux yeux du public un comportement que les nombreux témoins décrivirent comme « bestial », « indécent ». Tout à coup, après l'arrivée de sa visiteuse céleste, on voit la jeune voyante se débarrasser de son cierge, le donner à sa tante Bernarde, puis se déplacer rapidement sur les genoux d'un côté et de l'autre, de manière erratique. Elle gratte le sol, semblant chercher quelque chose. Elle patauge avec ses deux mains dans l'eau boueuse qui commençait à sourdre de la terre là où elle avait creusé, puis lape cette boue, arrache l'herbe alentour et la mange. Plusieurs fois, elle se déplace à genoux (on disait souvent, dans les témoignages, à quatre pattes) change de direction, l'air inquiet, vers la grotte puis vers le Gave. Enfin, elle revient à l'endroit où elle avait gratté le sol et se couvre le visage entier de cette boue. La foule perturbée commence à murmurer, pleine de dégoût, furieuse et frustrée de voir B dans une attitude évoquant bestialité et folie. Regrettant son diagnostic favorable, se sentant dupée, honteuse d'avoir cédé à ce qu'elle

17. Sœur Stanislas Paschal (1 ${ }^{\mathrm{er}}$ mai 1918), LHA, VI, p. 78.

18. Sœur Émile Marcillac, enquête préalable de 1907, LHA, VI, p. 78.

19. Amélie Gouteyron, déposition du 3 mars 1909, LHA, VI, p. 79. 
appelait maintenant "sa crédulité ». Une jeune fille, Elfrida Lacrampe, que le receveur des impôts Jean-Baptiste Estrade avait convaincue de venir assister à «la scène des visions de Bernadette " qu'il décrivait comme étant alors plus " belle que l'actrice Rachel ", s'écrie : "En fait de Rachel, tu nous as menés voir une petite merdeuse ! » La tante Bernarde intervient, essuie le visage de sa nièce, puis la gifle (publiquement). B retourne à sa place, termine son chapelet, se lève et, calmement, retourne chez elle, au milieu d'une foule hostile. De toutes parts, elle sera longuement interrogée sur la signification de tout cela. Elle répondra qu'elle avait agi sur les ordres d'Aquero qui lui avait demandé de trouver une source et de s'y laver. Ce n'est qu'un peu plus tard, avec les explications de B et les premières guérisons avec l'eau de cette source, que le mystère de ce comportement sera dévoilé aux dévots.

Cette scène décrite par les adeptes comme visuellement et moralement perturbante permet de réfléchir à l'importance du cadre esthétique et formel des apparitions mariales, à leur rapide mise en conformité avec les scènes qui, sur maints tableaux, les représentent et leur donnent un format scénique strict. On a ici, avec la lecture du visage de B par ceux qui l'entourent, une réponse à la question « de l'entrée dans l'image » posée au début de cet article. Le processus d'attestation, de mise en conformité se fait au long d'une série d'épreuves, au cours desquelles se perd l'indétermination de la situation, jusqu'à la fin du processus, la reprise en main de l'institution (y compris l'achat du site et son contrôle), et l'évacuation de la voyante hors de la scène. L'empathie ressentie pour la visionnaire par les personnes comprises dans le cercle de l'apparition, celles qui voient la voyante voir, leur attendrissement, dépend d'une grammaire esthétique qui doit être reconnaissable par tous. La rupture de ce schème rompt d'emblée la confiance en la visionnaire, et, partant, en sa vision. Dans cette scène publique, B, soudain, n'est plus transfigurée, mais défigurée, elle redevient Peau d'Ane et retourne à sa condition habituelle, la déconsidération.

\section{"Dire, sans mots, la situation politique " vs " faire de la politique " avec une apparition}

Se poser la question de l'expérience du surnaturel, tenter d'élaborer une généralisation possible du statut, des transformations, des ressorts et des ressources de cette expérience et des entités qu'elle meut, non pas en général, mais simplement dans les cas d'apparitions modernes et contemporaines au sein des univers marqués par le catholicisme, demande d'effectuer des détours par une description de séquences empiriques qui permettent en effet de suivre la modification des types d'acteurs engagés, noter leur «taille» ( "l'Église », «la Presse », «telle personne »), suivre la transformation des qualifications et des messages transmis, et les conflits entre " qualificateurs ». Il permet ici de circonscrire la transformation du message, souvent écrasé sous une seule forme : la critique conservatrice 
de la modernité. On voit plutôt ici à l'œuvre les processus complexes, détournés, indirects, imagés, par lesquels on passe, en changeant de porte-parole, de la critique locale de la modernisation par B et ses pairs, comme groupe social, à la critique générale et principielle de la modernité par certaines factions de l'Église et leurs relais. Résumons ces séquences et les médiations qu'elles mobilisent.

D'abord la jeune fille est surprise. C'est là un point sociologiquement essentiel car il configure une situation exprimant la non verbalisation comme telle. Cette scène de vision initiale configure par défaut la plainte infra-politique de la jeune $B$ auprès d'un porte-parole réparateur, implicitement compréhensif, aimant et silencieux. Le tableau vient exprimer les effets de la modernisation économique et politique (et ses effets de groupes et de personnes délaissés à tous égards) sur quelqu'un qui ne peut, via une quelconque plainte, l'analyser directement et globalement et encore moins agir. Elle voit seulement devant elle une forme humaine féminine animée, déférente, qu'elle appellera dans le procès-verbal de la commission d'enquête "une vision », terme qui sera raturé par le curé de la paroisse et auquel il substituera celui «d'apparition ».

Ensuite, malgré les obstacles du monde tel qu'il est ordonnancé, B se fera l'interlocutrice, la médiatrice, puis le relais de «cette forme ». Elle lui donnera les premiers linéaments d'une visibilité publique à l'intention du public restreint constitué par son monde à elle : les enfants pauvres du village, ses amies. Là, elle animera, donnera consistance objective, à une scène : un être liminaire rendant visite à des gens liminaires, dans un lieu liminaire, à une heure liminaire, dans une langue désormais liminaire. Plus tard enfin, après les épreuves d'identification et de nomination, lorsque l'Église par le biais de l'ordre lazariste prendra en main "le sanctuaire ", et alors que B est désormais traitée par le public des fidèles comme une vivante relique (on voulait toucher celle qui avait eu une relation visuelle et des échanges verbaux avec la Vierge), elle fut retirée du lieu. Celui-ci ne pouvait être saisi par deux polarités, et B fut emmenée sur décision des instances ecclésiastiques, loin, dans un couvent. Là, bien sûr, on ne manqua pas de lui rappeler qu'elle n'avait été qu'un "instrument ", qu'aucune "élection » personnelle n'avait été en jeu. Qu'elle ne devait tirer vanité de rien. Cependant, et contre sa volonté, on l'exhibait et lui faisait " rejouer la scène ", mais cette fois, pour le compte de l'institution, de son message anti-moderne, et en son nom. Puis il y eut un nouveau retournement de situation qui redonna à $\mathrm{B}$ une place, une posture, contrôlée par l'Église, mais laissant percevoir quelque chose de son message initial : la force critique des perturbateurs de hiérarchie. À sa mort, il fallut à nouveau que l'Église modifie cette identité d' "instrument " en vue d'enjeux de canonisation. Il fallut redonner à la future "Sainte ", consistance et volonté, et refaire d'elle quelqu'un qui avait été choisi, qui avait été la partenaire active de sa vision, ou de son apparition. Dans le même temps, cependant, des partis politiques (les ultramontains) s'associeront à la tendance de l'Église qui s'exprimait à Lourdes, pour transformer le message initial d'une 
plainte sociale contre les effets d'un type de modernisation pour la transformer en guerre conservatrice contre la modernité. C'est dans cette série de transformations, de traductions, de trahisons qui parcourent des espaces qui vont de l'intime au collectif et au public, que se déploie ici, me semble-t-il, comme une espèce de joker prêt à toutes les alliances, l'expérience du surnaturel. Ainsi, le sens politique, social et religieux donné par les divers médiateurs ou porte-parole de " ce qui apparaît » varie et se transforme au cours du processus de nomination, engageant des relations à chaque fois différentes au surnaturel et à ses référents imaginaires, émotionnels, sociaux et politiques. De multiples sentiments intimes et collectifs sont mobilisés dans ces processus, qui permirent de construire un objet: un lien de conséquence entre deux sphères jusque-là séparées. Par ces biais, est en effet constitué une relation forte entre la sphère de l'intime (ce à quoi l'on est attaché ici et maintenant et qui constitue la trame de l'expérience quotidienne, des siens, de son monde) et du politique (vu comme l'ensemble des modalités institutionnelles de sa préservation, ou de sa transformation). C'est alors, par un exercice de montée en généralité que ces configurations d'attachements sont confiées au politique. On comprend mieux alors pourquoi, lorsqu'il corrige le procès-verbal des déclarations de Bernadette devant le commissaire de police, l'abbé Peyramale raye le mot «vision » et le remplace par celui «d'apparition ", objet sémantiquement moins spéculatif et plus maniable, et qui ouvre à l'entité la perspective d'alliances d'un type nouveau.

\author{
Élisabeth CLAVERIE \\ CNRS-EHESS - Institut Marcel Mauss \\ claverie@ehess.fr
}

\title{
Bibliographie
}

Apolito Paolo, 1998, Apparitions of the Madonna at Olivieto Citra: Local Visions and Cosmic Drama, Philadelphia, The Pennsylvania State University Press.

BERTRIN Georges, 1905, Histoire critique des événements de Lourdes, apparitions et guérisons, Bruxelles, Union des CEuvres de Presse Catholique.

Blackbourn David, 1994, Marpingen, Apparitions of the Virgin Mary in NineteenthCentury German Village, New York, Alfred A. Knopf.

Carel Alexis, 1949, Le Voyage de Lourdes, Paris, Plon.

CHARcot Jean-Martin, 1897, La Foi qui guérit, Paris, Félix Alcan.

ChARUTy Giordanna, 1992, Le Couvent des Fous. L'internement et ses usages en Languedoc aux XIX et XX siècles. Paris, Flammarion.

-, 1988, «Le psychiatre et ses “autres », Ethnologie française, 18-2, pp. 158-165.

Christian William Jr., 1996, Visionaries: The Spanish Republic and the Reign of Christ, Berkeley, University of California Press.

ClaverIE Élisabeth, 2003, Les Guerres de la Vierge. Une anthropologie des apparitions, Paris, Gallimard.

-, 2008, Le monde de Lourdes, Paris, Gallimard, coll. « Découvertes ». 
Harris Ruth, Hieaux-Heitzmann Marie-Lise, 1999, Lourdes, La grande histoire des apparitions, des pèlerinages et des guérisons, Paris, Jean-Claude Lattès.

HuYsmans Joris Karl, 1989 [1906], Les Foules de Lourdes, Grenoble, Jérôme Million.

LATOUR Bruno, 1990, "Quand les anges deviennent de bien mauvais messagers ", Terrain, 14, pp. 76-91.

PolanyI Karl, 1983, La Grande Transformation. Aux origines politiques et économiques de notre temps, Paris, Gallimard.

Taves Ann, 1999, Fits, Trances and Visions: Experiencing Religion and Explaining Experience from Wesley to James, Princeton, Princeton University Press.

Werfel Franz, 1953, Le Chant de Bernadette, Paris, Albin Michel.

Zola Émile, 1995 [1894], Lourdes, Paris, Folio-Gallimard.

\section{Résumé}

Cet article traite, s'agissant des apparitions de Lourdes, en France, en 1858, du long processus d'identification opéré par une série d'acteurs individuels et collectifs, de "ce qui apparaît" à une jeune fille du lieu. Le sens politique, social et religieux donné par les divers médiateurs ou porte-parole, varie et se transforme au cours du processus de nomination, engageant alors des relations diverses au surnaturel et à ses référents imaginaires et politiques. De multiples émotions intimes et collectives sont mobilisées dans ce processus, qui permettent de construire un lien de conséquence fort entre l'intime (ce à quoi on est attaché, les siens, son monde) et le politique (les modalités étatiques de sa préservation, ou de sa transformation) à qui ce lien est confié.

Mots-clés : Apparitions, Lourdes, Voyants, visionnaires, religion et politique, émotions, surnaturel, expérience.

\section{Abstract}

The paper is about Marian apparitions in Lourdes (France) in 1858. It focuses on the different ways by which the entity that appears is given a name by different actors through a long process. In this process of appropriation, many different mediators are expressing different social, emotional and political views. All along this process, links are created between the intimacy of the individual or collective groups (what they are attached to) and politics (the enforced ways these attachments can be protected or changed).

Key words: Marian apparitions, Lourdes, visionnaries, religion and politics, supernatural, experience.

\section{Resumen}

Este articulo trata, a partir de las apariciones de Lourdes, en Francia, en 1858, del largo proceso de identificación operado por una serie de actores individuales y colectivos, de "aquello que aparece" a una joven del lugar. El sentido politico, social y religioso dado por los distintos mediadores o voceros varía y se transforma durante el curso del proceso de nominación, implicando entonces distintas relaciones de lo 
I 28 - ARCHIVES DE SCIENCES SOCIALES DES RELIGIONS

sobrenatural y de sus referentes imaginarios y politicos. Múltiples emociones intimas $y$ colectivas se movilizan en este proceso, y permiten construir una relación de consecuencia fuerte entre lo intimo (aquello a lo que estamos ligados, los nuestros, nuestro mundo) y lo politico (las modalidades estatales de su preservación, o de su transformación) a quien este lazo es confiado.

Palabras clave: Apariciones, Lourdes, videntes, visionarios, religión y política, emociones, sobrenatural, experiencia. 